\title{
Chromosome Analysis of in vitro Induced Androgenetic Haploids of Nicotiana tabacum
}

\author{
B. Dimitrov, P. Popov ${ }^{1}$ and N. Zagorska \\ Institute of Genetics, Bulgarian Academy of Sciences, Sofia, Bulgaria
}

Received April 26, 1980

Data about morphology of chromosomes of $N$. tabacum are met in some works dealing with problems of genetics and cytogenetics of this species (Kostoff 1943, Goodspeed 1954). Because of the comparatively large number of chromosomes diploid $(2 \mathrm{n}=48)$, the relatively small size and the extremely similar morphology of many of the chromosome pairs a more profound study of the karyotype and the idiotype of this species is lacking. These difficulties could be avoided if haploids are used, this allows a more detailed morphological characteristics of each individual chromosome to be made.

The achievements in inducing haploids in vitro in recent years, the greatest being undoubtedly with $N$. tabacum, made it possible to apply the method of tissue cultures in studying chromosomes structure and morphology.

The aim of this work is to make a more detailed study of the karyotype of $N$. tabacum using in vitro induced androgenetic haploids on the basis of the biometric characteristics of the centromeric position of the individual chromosomes obtained by the numerical values of the arm ratio ( $r$ ) and the centromeric index (i) according to the nomenclature of the centromeric position proposed by Levan et al. (1964).

\section{Material and methods}

For the purposes of the study anthers of $N$. tabacum cultivar Burlley 21, were used, namely anthers containing pollen in the mononuclear stage or in the first mitosis stage only. The stage of development of anthers is determined by means of squash preparations stained in $4 \%$ acetocarmine. Before being put in a nutrient medium the anthers were sterilized for 5 minutes in a $7 \%$ filtered solution of calcium hypochloride. Then, they were washed three times in distilled water kept for one hour in an autoclave under a pressure of 2 atmospheres.

The sterile anthers were put in nutrient medium of Nitsch (1969) with caseine hydrolysate $1 \mathrm{~g} / \mathrm{l}$, mesoinosite $10 \mathrm{~g} / \mathrm{l}$, kinetine $0.1 \mathrm{mg} / \mathrm{l}$, IAA $0.1 \mathrm{mg} / \mathrm{l}$, sucrose $20 \mathrm{~g} / \mathrm{l}$ and agar $8 \mathrm{~g} / \mathrm{l}$ added to it. The medium had a $\mathrm{pH}$ value of 5.5. Sterilization was done for 20 minutes at $120^{\circ} \mathrm{C}$.

The anther cultures were grown under the conditions of 16-hour light periods, additional lighting being provided by luminescent lamps with an intensity of $300 \mathrm{~lx}$ at a temperature of $26^{\circ} \mathrm{C}$ and relative humidity $80 \%$.

Root formation of the plants obtained proceeded in White medium in which

\footnotetext{
1 The University of Plovdiv "Paisii Hilendarski”, Plovdiv, Bulgaria
} 
$2 \mathrm{ml} / \mathrm{l}$ of ferulic acid were added.

The plants were then planted in pots with soil and were grown under greenhouse conditions at $20-22^{\circ} \mathrm{C}$.

The cytological studies of the number and morphology of chromosomes were made by the standard squash method. The cytological preparations were made from root meristems previously treated in $0.025 \%$ colchicine and fixed in alcoholacetic acid for $24 \mathrm{hrs}$. The material was stained with 1: 1 mixture of Schiff and $4 \%$ acetoorceine.

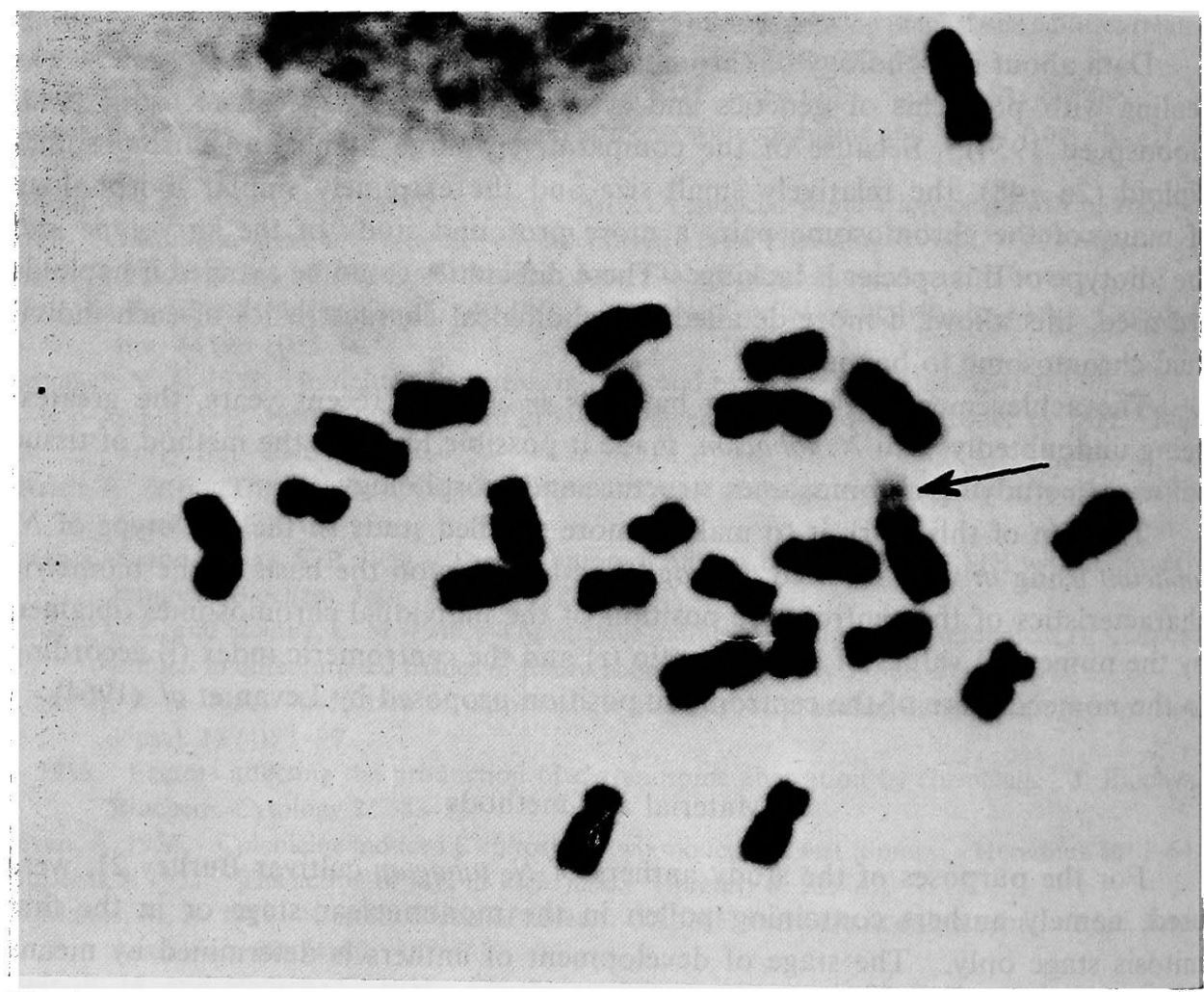

Fig. 1. Metaphase of haploid N. tabacum. SAT-chromosome indicated with dark arrow. $\times 2500$.

For the purposes of studying the morphology of the individual chromosomes 20 metaphase plates, most suitable to this end, were photographed (Fig. 1) and on this basis karyograms were plotted. For each chromosome the following values were measured and calculated:

-Arm ratio ( $r$ ) calculated by the formula $\mathrm{r}=1 / \mathrm{s}$, where 1 - long arm of chromosome and s-short arm of chromosome;

- Centromeric index (i) calculated by the formula $i=100 /(r+1)$;

-Absolute length of metaphase chromosome $\left(\mathrm{L}_{\mathrm{abs}}\right)$.

The data obtained were processed by variational statistics analysis according to Student-Fisher's method. The average values of $r$ and $L_{a b s}$ of each chromosome 
Table 1. Mean values of the arm ratio ( $r$ ) and centromeric index (i) for individual chromosomes and chromosome groups

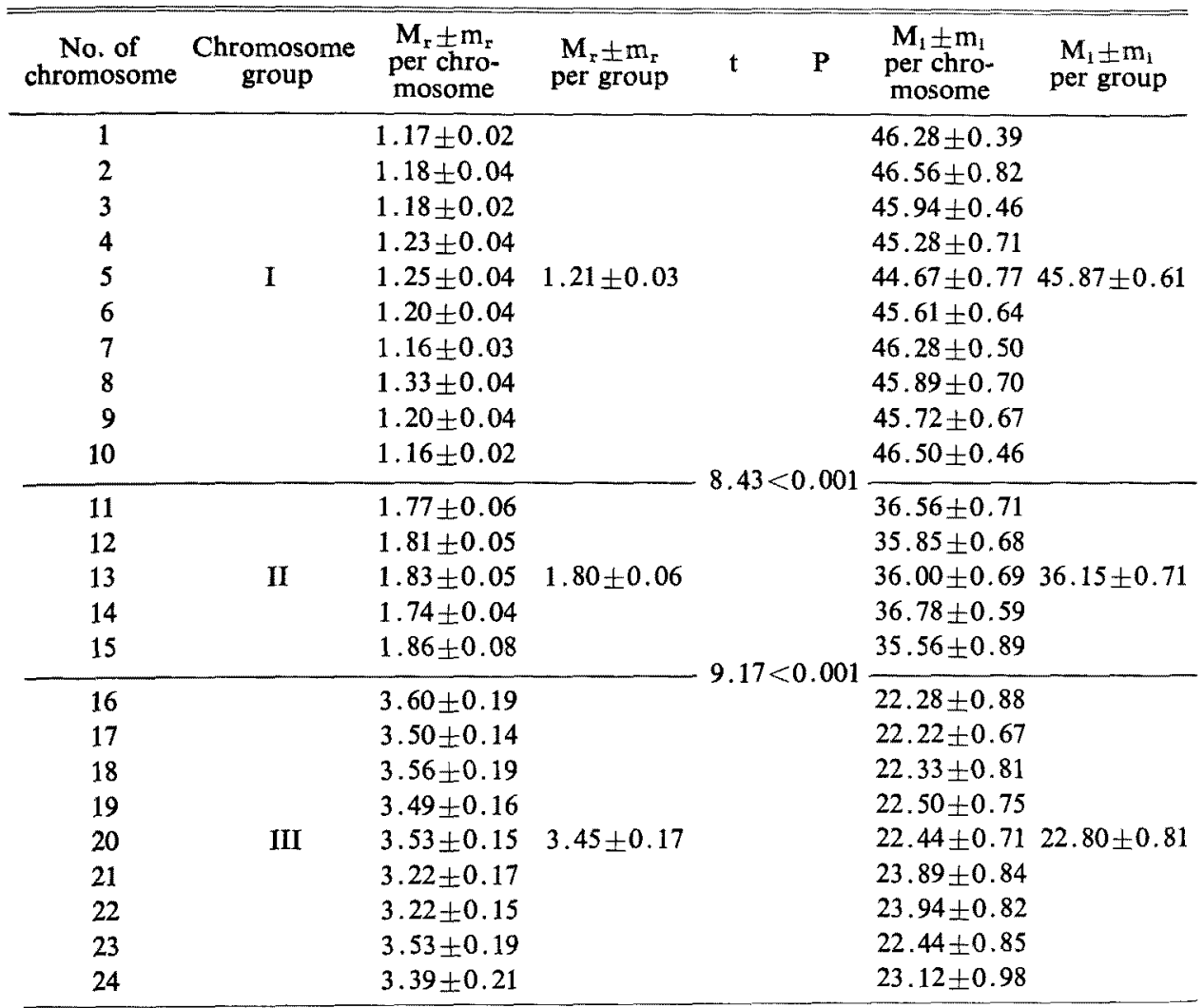
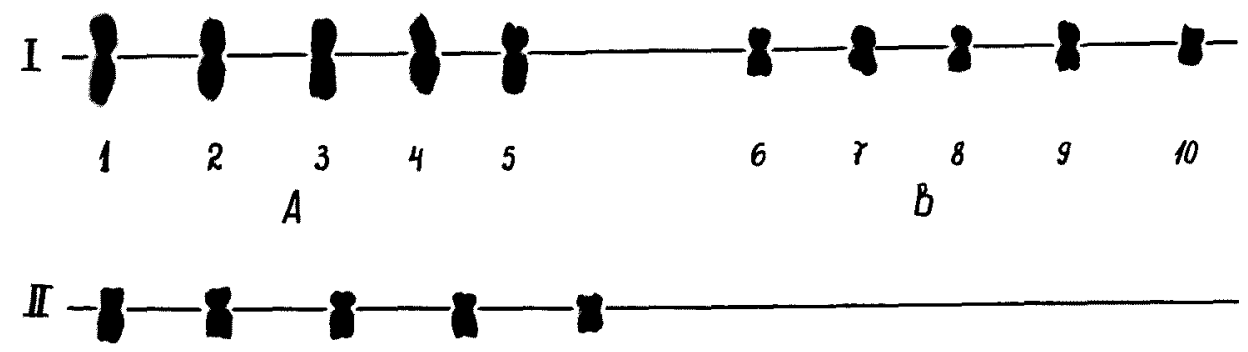
$\begin{array}{lllll}11 & 12 & 13 & 14 & 15\end{array}$

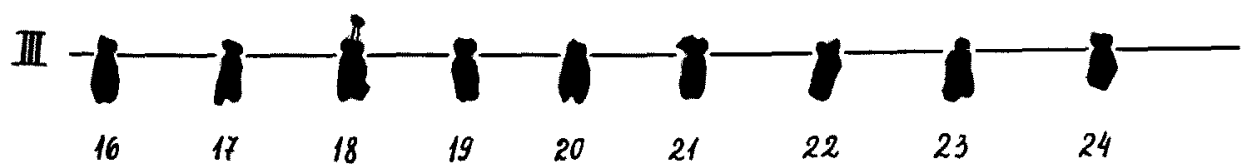

Fig. 2. Karyotype of N. tabacum. 
were used to calculate the set of equations

$$
\begin{aligned}
& x+y=L_{a b s} \\
& x / y=r
\end{aligned}
$$

The resulting data were used to plot the idiotype.

\section{Results and discussion}

The average values from the biometrical analysis of the individual chromosomes are an indication that the karyotype of the haploid studied by us consists of 24 chromosomes. Morphologically, they can be divided in the following groups and sub-groups (Figs. 2 and 3, and Table 1):

Group I. It covers 10 chromosomes with a median centromeric position and values of arm ratio and centromeric index average for the group, $r=1.21 \pm 0.03$ and $\mathrm{i}=45.87 \pm 0.61$ respectively. Depending on the absolute lengths of the metaphase chromosomes two subgroups could be formed (Figs. 2 and 3), i.e.:

Sub-group A-consisting of 5 metacentric chromosomes of larger size;

Sub-group B-consisting of 5 metacentric chromosomes of smaller size.

The variational statistics analysis of the absolute lengths of the individual chromosomes in this group reveals a significant difference between the average length of the smallest chromosome of sub-group A and the largest chromosome of sub-group B. This allows the formation of these two chromosome sub-groups within the group of the metacentric chromosomes (Table 2). The differences in the lengths make the chromosomes in these two sub-groups easily distinguished among themselves (Fig. 2).

Group II. It consists of 5 chromosomes with sub-median centromeric position and values of arm ratio and centromeric index average for the group, $r=1.80 \pm 0.06$ and $i=36.15 \pm 0.71$ respectively. The absolute lengths of the chromosomes in this group are close thus making their differentiation difficult to a large extent. However, the sub-median position of the centromeres makes them quite well distinguished from the remaining chromosomes in the haploid metaphase of $N$. tabacum studied by us (Tables 1 and 2 and Figs. 2 and 3).

Group III. It consists of 9 chromosomes with centromeric sub-terminal position and with values of arm ratio and centromeric index average for the group, $r=3.45 \pm 0.17$ and $i=22.80 \pm 0.81$ respectively. In the third chromosome of this group we found a secondary constriction located upon the short arm which separates a satellite (Figs. 1 and 2). In the various metaphases studied it is located at different distances from the chromosome body and remains linked to it through barely visible chromatin fibers. In earlier cytological studies of $N$. tabacum's karyotype the presence of similar satellites on other chromosomes has been established (Goodspeed 1954). However, none of the metaphases studied by us revealed any secondary constrictions and satellites located on any other chromosome than the third chromosome of group 3. This gives us grounds to suppose that this chromosome is the only SAT-chromosome in the karyotype of N. tabacum and that the other SAT-chromosomes mentioned by Goodspeed (1954) are in all proba- 
Table 2. Mean values of the absolute length of individual chromosomes and chromosome groups and sub-groups

\begin{tabular}{|c|c|c|c|c|c|c|}
\hline $\begin{array}{l}\text { No. of } \\
\text { chromosome }\end{array}$ & $\begin{array}{l}\text { Chromosome } \\
\text { group }\end{array}$ & Subgroup & $\mathbf{M}_{\mathrm{LabB}} \frac{ \pm}{\mathrm{m}_{\mathrm{LB} B s}}$ & $\mathbf{P}$ & $\begin{array}{c}\mathrm{M}_{\mathrm{Labs}} \pm \\
\mathrm{m}_{\mathrm{Labs}} \\
\text { per chromo- } \\
\text { some } \\
\text { subgroup } \\
\text { in } \mu \mathrm{m}\end{array}$ & $\begin{array}{l}\mathrm{M}_{\mathrm{Lgbs}} \pm \\
\mathrm{m}_{\mathrm{Labs}} \\
\text { per chromo- } \\
\text { some } \\
\text { group } \\
\text { in } \mu \mathrm{m}\end{array}$ \\
\hline 1 & & & $6.11 \pm 0.23$ & \multirow{10}{*}{$4.78<0.001$} & & \\
\hline 2 & & & $5.87 \pm 0.22$ & & & \\
\hline 3 & & A & $5.58 \pm 0.24$ & & $27.81 \pm 1.11$ & \\
\hline 4 & & & $5.36 \pm 0.21$ & & $(26.95 \%)$ & \\
\hline 5 & & & $4.89 \pm 0.21$ & & & $44.12 \pm 1.83$ \\
\hline 6 & & & $3.60 \pm 0.14$ & & & $(42.75 \%)$ \\
\hline 7 & & & $3.49 \pm 0.16$ & & $16.31 \pm 0.72$ & \\
\hline 8 & & B & $3.22 \pm 0.10$ & & $(15.81 \%)$ & \\
\hline 9 & & & $3.13 \pm 0.21$ & & & \\
\hline 10 & & & $2.87 \pm 0.11$ & & & \\
\hline 11 & \multirow{5}{*}{ II } & & $4.59 \pm 0.23$ & & & \multirow{5}{*}{$\begin{array}{c}19.22 \pm 0.78 \\
(18.63 \%)\end{array}$} \\
\hline 12 & & & $4.11 \pm 0.18$ & & & \\
\hline 13 & & & $3.73 \pm 0.13$ & & & \\
\hline 14 & & & $3.56 \pm 0.14$ & & & \\
\hline 15 & & & $3.23 \pm 0.10$ & & & \\
\hline 16 & \multirow{9}{*}{ III } & & $4.93 \pm 0.17$ & & & \multirow{9}{*}{$\begin{array}{c}39.87 \pm 1.35 \\
(38.63 \%)\end{array}$} \\
\hline 17 & & & $4.73 \pm 0.13$ & & & \\
\hline 18 & & & $4.63 \pm 0.13$ & & & \\
\hline 19 & & & $4.56 \pm 0.14$ & & & \\
\hline 20 & & & $4.49 \pm 0.15$ & & & \\
\hline 21 & & & $4.36 \pm 0.16$ & & & \\
\hline 22 & & & $4.31 \pm 0.18$ & & & \\
\hline 23 & & & $4.09 \pm 0.17$ & & & \\
\hline 24 & & & $3.77 \pm 0.17$ & & & \\
\hline
\end{tabular}

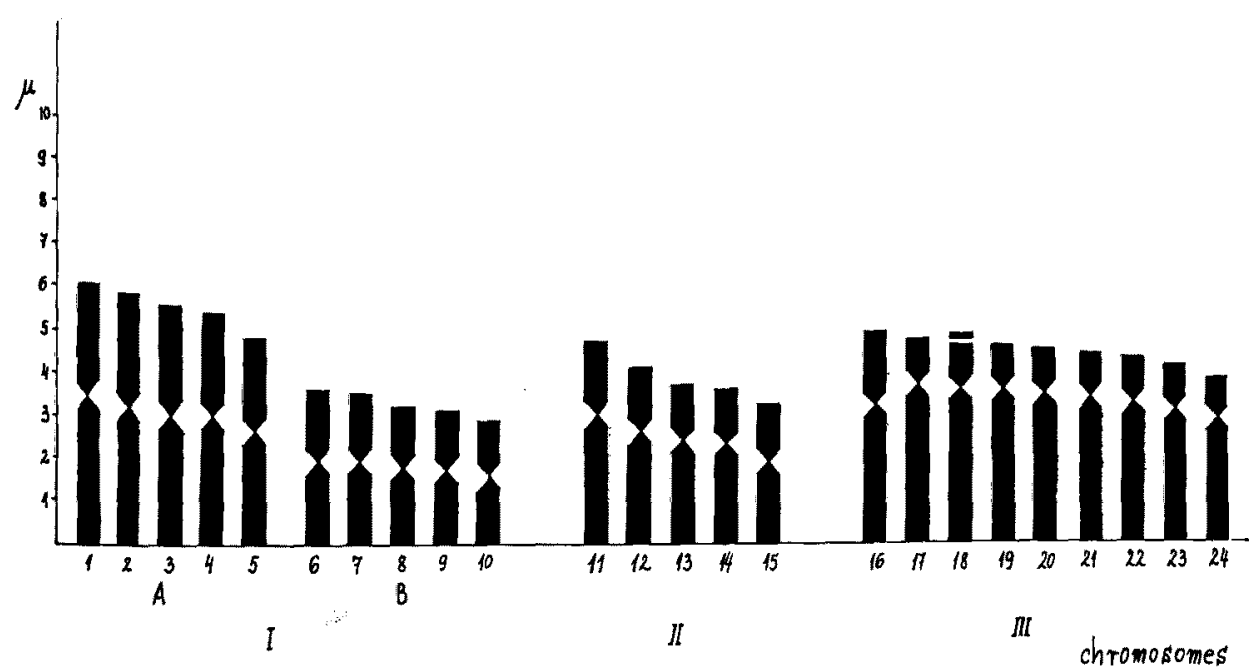

Fig. 3. Idiotype of N. tabacum. 
bility the result of the imperfection of the methods of karyological studies at that time and the inexact arrangement of the SAT-chromosomes in the different karyograms.

The studies of the morphology of this chromosome did not take into consideration the size of the satellite and its distance from the chromosome body.

As seen from Table 1, both the average values of arm ratio and centromeric index which characterize the centromeric position in the different chromosome groups and the values of these indices for each chromosome coincide entirely with the nomenclature of the centromeric position proposed by Levan et al. (1964). This fact gave us reasons to state that the arranging of the chromosomes of $N$. tabacum into different morphological groups and sub-groups has been correct and advisable.

The results from the measuring of the individual chromosome are an indication that the average values of the absolute lengths of the chromosomes in the cells of the haploid studied by us are in the range from $6.11 \pm 0.23 \mu \mathrm{m}$ to $2.87 \pm 0.11 \mu \mathrm{m}$ (Table 2). Longest in the karyotype is the first chromosome of the sub-group of large metacentrics, and shortest is the one of the sub-group of small metacentrics. The average total length of the metaphase chromosomes amounts to $103.21 \pm 3.96$ $\mu \mathrm{m}$, the various chromosome groups and sub-groups occupying different parts of it.

The group of metacentric chromosomes occupies $42.75 \%$ of the total length $(44.12 \pm 1.83 \mu \mathrm{m})$, of which $26.95 \%(27.81 \pm 1.11 \mu \mathrm{m})$ are occupied by the subgroups of the large metacentrics and $15.81 \%(16.31 \pm 0.72 \mu \mathrm{m})$ by the small metacentrics. The group of sub-metacentric chromosomes has a total length of $19.22 \pm$ $0.78 \mu \mathrm{m}$ and occupies barely $18.63 \%$ of the total length of the metaphase chromosomes in the haploid complement. The group of sub-telocentric chromosomes has an overall length of $39.87 \pm 1.35 \mu \mathrm{m}$ and occupies $38.63 \%$ of the total length. When measuring and calculating the absolute lengths of the individual chromosomes we did not consider the size of the satellite and its distance from the chromosome body.

The largest chromosome in the karyotype of the haploid of $N$. tabacum occupies $5.92 \%$ of the total length of the metaphse chromosomes, and the smallest chromosome $-2.78 \%$.

We did not find any data in previous publications about the absolute length of the chromosomes of $N$. tabacum for which reason we could not make any comparisons and closer interpretation of our results.

\section{Summary}

The metaphase chromosomes of the haploid of $N$. tabacum under study can be divided in 3 groups, the first one being composed of two sub-groups depending on the centromeres position and the absolute length of the individual chromosomes. The third chromosome of the group of the sub-telocentric chromosomes has a satellite and can serve as a marker chromosome. The longest chromosome in the karyotype is that of the sub-group of large metacentric chromosomes while the shortest chromosome is the last one of the sub-group of small metacentric chromosomes. The highest percentage participation in the total length of the metaphase 
chromosomes is that of the group of metacentric chromosomes $(42.75 \%)$, the lowest one being that of the group of sub-metacentric chromosomes $(18.63 \%)$.

\section{References}

Goodspeed, T. H. 1954. The genus Nicotiana. Chronica Botanica, Waltham, Mass., U.S.A.

Kostoff, D. 1943. Cytogenetics of the genus Nicotiana. Karyosystematics, genetics, cytology, cytogenetics and phylesis of tobaccos. State Printing House, Sofia 1941-1943.

Levan, A., Fredga, K. and Sandberg, A. A. 1964. Nomenclature for centromerics position on chromosomes. Hereditas 52: 201-220.

Nitsch, J. P. 1969 . Experimental androgenesis in Nicotiana. Phytomorphology 19: 389-404. 